\section{Religião e transtornos mentais em pacientes internados em um hospital geral universitário}

\author{
Religion and psychiatric disorders in patients \\ admitted to a university general hospital
}

\footnotetext{
${ }^{1}$ Faculdade de Ciências Médicas, Universidade Estadual de Campinas, Campinas, Brasil.

Correspondência P. Dalgalarrondo Departamento de Psicologia Médica e Psiquiatria Faculdade de Ciências Médicas, Universidade Estadual de Campinas. C. P. 6111, Campinas, SP 13083-970, Brasil. pdalga@fcm.unicamp.br
}

\begin{abstract}
In order to evaluate the prevalence of psychiatric disorders in a Brazilian general hospital and their association with religious denomination and religiosity, 253 inpatients were interviewed. A socio-demographic questionnaire and an instrument for diagnosis of mental disorders (MINI-Plus) were applied. Distribution of religious denominations was: Catholic $63.2 \%$ ( $n$ $=177)$, Evangelical Protestant 20.4\% ( $n=57)$, Spiritist 4.3\% ( $n=12)$, traditional Protestant $2.3 \%(n=8)$, and "no religion" $7.5 \%(n=21)$. Degree of religiosity was: very religious $43.2 \%$ ( $n=$ $116)$, religious $46.9 \%(n=129)$, hardly religious 9.8\% ( $n=27)$, and not at all religious $1.1 \%$ ( $n=$ 3). Evangelical (Pentecostal) religious affiliation and frequent attendance at worship services were associated with fewer alcohol problems. Membership in an Evangelical (Pentecostal) church may thus have an inhibitory effect on alcohol dependence or abuse. Intensity of religiosity was moderately associated with overall prevalence of disorders, especially bipolar disorder. It is reasonable to conclude that extreme situations (very intense versus very limited religious participation) are related to this finding, associating both an exacerbated pursuit of religion and alienation from it with altered mental states.
\end{abstract}

Religion; Mental Disorders; Inpatients

\author{
Rachel Esteves Soeiro ${ }^{1}$ \\ Elisabetta S. Colombo 1 \\ Marianne H. F. Ferreira 1 \\ Paula S. A. Guimarães 1 \\ Neury J. Botega 1 \\ Paulo Dalgalarrondo 1
}

\section{Introdução}

A relação entre religião e a prevalência dos principais transtornos mentais tem sido um tópico de crescente interesse científico 1. Embora a maioria dos estudos, principalmente norte-americanos, aponte para uma possível ação protetora da religiosidade sobre a saúde mental ${ }^{2}$, dados da realidade brasileira são ainda relativamente escassos. Recentemente, Botega et al. ${ }^{3}$ identificaram numa amostra de 515 pessoas representativas da população de Campinas, São Paulo, Brasil, que pertencer a denominações evangélicas associa-se a menor freqüência de uso problemático de álcool, porém a pertença a igrejas evangélicas associou-se a uma freqüência maior de sintomas depressivos; e ser espírita kardecista associou-se à freqüência maior de problemas na área de saúde mental 4.

Em relação à população com transtornos físicos, crenças e práticas religiosas mais intensas foram associadas a menores taxas de sintomas depressivos em mulheres idosas com fratura de bacia 5 e à freqüência menor de depressão em homens idosos internados por doenças não-psiquiátricas ${ }^{6}$. Em pacientes hospitalizados com doenças físicas, ter maior envolvimento religioso implica menor propensão a desenvolver sintomas depressivos, e, quando tais sujeitos ficam deprimidos, recuperam-se mais rapidamente 7,8 . Na mesma linha, pacientes com câncer ou doença de Alzheimer tendem a adaptar-se melhor 
a suas limitações, quando mais envolvidos com a religião ${ }^{8}$.

Estudos realizados no Brasil mostram que pacientes internados por transtornos psiquiátricos pertencentes a grupos evangélicos têm uma maior freqüência de diagnósticos de psicoses 9. Jovens evangélicos apresentam menor uso de álcool e drogas e menos sintomas psiquiátricos do que jovens católicos e espíritas 10. Além disso, pacientes que tiveram uma criação religiosa mais intensa na infância parecem envolver-se menos com o uso de álcool ou drogas do que aqueles sem tal educação religiosa 10. Por outro lado, estudos recentes em nosso meio têm indicado que pertencer a religiões minoritárias (como igrejas evangélicas pentecostais e espiritismo kardecista) pode associar-se à pior saúde mental 11 e à pior qualidade de vida, talvez refletindo um processo social de busca de novos agrupamentos religiosos por pessoas em situação de sofrimento 12 .

\section{Objetivo}

O presente estudo tem como objetivo determinar a prevalência de transtornos mentais em pacientes internados em um hospital geral universitário. Também visa identificar se tal prevalência é diferente entre pacientes com ou sem religião e/ou com diferentes níveis de religiosidade.

\section{Pacientes e métodos}

Este é um estudo transversal que foi realizado nas enfermarias do Hospital das Clínicas da Universidade Estadual de Campinas (UNICAMP). Trata-se de hospital geral universitário terciário com 750 leitos, cujos serviços cobrem uma área de cerca de 2,5 milhões de habitantes.

\section{Pacientes}

O tamanho amostral foi calculado após a realização de um estudo-piloto, estimando-se em $30 \%$ a prevalência de transtornos mentais. Entre junho de 2003 e janeiro de 2004, 304 pacientes foram selecionados dentre um total de 898 pacientes internados. Nesse período, todas as internações foram numeradas consecutivamente e os pacientes foram selecionados com base numa tabela de números aleatórios. Os critérios de inclusão foram: pacientes maiores de 18 anos, provenientes de enfermarias cirúrgicas e clínicas (exceto a de psiquiatria), internados há pelo menos 48 horas e que apresentassem compreensão satisfatória da entrevista. Após as entrevistas com os pacientes selecionados, 31 foram excluídos (15 estavam desorientados ou confusos, seis pacientes estavam internados a menos de 48 horas, cinco pacientes eram menores de 18 anos e cinco foram considerados incapacitados para responder às questões). Dos 273 indivíduos que preencheram os critérios, 253 pacientes concordaram em participar do estudo. O grupo de 51 pacientes não participantes (31 exclusões e 20 recusas) foi similar aos pacientes participantes em características sóciodemográficas e patologias clínicas.

\section{Instrumentos}

Utilizou-se um questionário sócio-demográfico elaborado especificamente para esta pesquisa, no qual a variável religiosidade foi representada pelos pacientes entrevistados de acordo com sua fé em Deus e atuação dentro de sua comunidade religiosa e um instrumento para diagnóstico de transtornos mentais, o Mini International Neuropsychiatric Inteview (MINI-Plus) 13. O MINI-Plus é uma entrevista padronizada breve, de duração em torno de 25 a 45 minutos, compatível com os critérios do Manual Diagnóstico e Estatístico de Transtornos Mentais - 4a Edição (DSM-IV) e da Classificação Estatística Internacional de Doenças e Problemas Relacionados à Saúde - 10a Revisão (CID-10). Esse instrumento diagnóstico foi validado no Brasil por Amorim 13 e revelou resultados referentes à confiabilidade bastante satisfatórios, apresentando sensibilidade $\geq 0,64$, especificidade $\geq 0,71$, valor preditivo negativo $\geq 0,84$ e valor preditivo positivo $\geq 0,6$. A fim de atender aos objetivos da presente pesquisa, foram selecionados os seguintes módulos: episódio depressivo maior, transtorno distímico, risco de suicídio, episódio maníaco e hipomaníaco, transtorno de pânico, agorafobia, fobia social, transtorno obsessivo compulsivo, transtorno de estresse pós-traumático, dependência e abuso de álcool, dependência e abuso de substância (não álcool), síndrome psicótica e transtorno de humor com características psicóticas, bulimia nervosa e transtorno de ansiedade generalizada.

\section{Procedimentos}

A todos os pacientes que preencheram os critérios de inclusão descritos, foi aplicado inicialmente o termo de consentimento informado e posteriormente os questionários sócio-demográficos e o MINI-Plus. As entrevistas foram realizadas à beira do leito, individualmente. $\mathrm{O}$ treinamento dos entrevistadores envolveu lei- 
tura do instrumento, entrevistas-piloto, e discussão com auxílio e supervisão de um docente do departamento de psiquiatra e co-autor deste trabalho (P.D.).

A análise estatística foi feita pelos programas Epi Info (Centers for Disease Control and Prevention, Atlanta, Estados Unidos) e SAS (SAS Inst., Cary, Estados Unidos). Para descrever o perfil da amostra segundo as variáveis em estudo, foram feitas tabelas de freqüência das variáveis categóricas, com os valores de freqüência absoluta (n) e percentual (\%), e estatísticas descritivas (com medidas de posição e dispersão - média, desvio padrão, valores mínimo, máximo e mediana) das variáveis contínuas.

Para analisar a associação entre duas variáveis categóricas, foram utilizados os testes qui-quadrado ou exato de Fisher (para valores esperados menores que cinco). Para comparar as variáveis numéricas entre dois grupos foi utilizado o teste de Mann-Whitney.

Para analisar a relação entre os fatores de interesse e a presença de transtorno mental, foi utilizada a análise de regressão logística, modelo logito. Foram feitas as análises univariada e multivariada com critério stepwise de seleção de variáveis.

O nível de significância adotado para os testes estatísticos foi de $5 \%(\mathrm{p}<0,05)$.

\section{Aspectos éticos}

A pesquisa envolveu pacientes maiores de dezoito anos, que apresentaram condições físicas e mentais suficientes para a aplicação dos questionários. Este estudo foi aprovado pelo Comitê de Ética em Pesquisa da Faculdade de Ciências Médicas da UNICAMP. Os pesquisadores comunicaram ao médico responsável casos de problema grave de transtorno mental ou de risco de suicídio.

\section{Resultados}

A média de idade foi de 47,1 anos $\pm 19,0$ anos (18-85), com $56,9 \%$ de homens $(n=144)$ e $43,1 \%$ de mulheres $(\mathrm{n}=109)$. A escolaridade média (em anos de escola cursados com sucesso) foi de 6,2 \pm 7,0 (0-25). A distribuição das denominações religiosas foi: católicos $62,8 \%(\mathrm{n}=159)$, evangélicos (pentecostais e históricos) 23\% ( $\mathrm{n}=58)$, espíritas 5,9\% ( $\mathrm{n}=15)$, e sem religião $7,9 \%(n=20)$. Consideraram-se muito religiosos $41,6 \%$ ( $n=104)$, religiosos $47,2 \%(n=118)$, pouco religiosos $10 \%(\mathrm{n}=25)$, e não religiosos $1,2 \%$ $(\mathrm{n}=3)$. Em relação à freqüência à Igreja, a média encontrada foi de 4,5 vezes por mês $\pm 9,0$.
Encontrou-se uma prevalência de 60\% (n = 152) para pelo menos um transtorno mental, de $29,2 \%(n=74)$ para transtorno depressivo maior, de $23,7 \%(n=60)$ para agorafobia, de $22,9 \%$ ( $\mathrm{n}=58)$ para risco de suicídio, de $15 \%(\mathrm{n}=38)$ para transtorno de ansiedade generalizada, de $7,9 \%(n=20)$ para transtorno bipolar, de $6 \%$ ( $\mathrm{n}=15$ ) para dependência de álcool, e de 3,2\% ( $\mathrm{n}=8)$ para abuso de álcool.

Não se verificou qualquer associação significativa entre a freqüência de diagnóstico de transtornos de ansiedade e as variáveis de religião e religiosidade.

Nas Tabelas 1, 2 e 3, são apresentadas as associações entre os diagnósticos supracitados (exceto transtornos de ansiedade) e suas relações com variáveis de religião e religiosidade.

$\mathrm{Na}$ Tabela 4, verificou-se que pessoas pouco religiosas ou sem religião tiveram cerca de sete vezes mais chance de ter algum diagnóstico de abuso ou dependência de álcool do que pessoas muito religiosas. Pessoas com pouca ou sem religiosidade tiveram cerca de seis vezes mais chance de apresentar transtorno bipolar do que quem é religioso. Pessoas muito religiosas em relação às que são regularmente (ou moderadamente) religiosas tiveram cerca de três vezes mais chance de ter o diagnóstico de transtorno bipolar.

\section{Discussão}

Por ter como principal foco pacientes clínicos internados em um hospital geral universitário, o presente trabalho gerou dados que não devem ser generalizados para a população geral. Os resultados obtidos dizem respeito, portanto, a uma população clínica internada e é nesse contexto que devem ser interpretados e, eventualmente, generalizados.

Os dados obtidos mostram, globalmente, que a filiação religiosa em uma denominação específica (evangélico, católico ou espírita) não foi um fator claramente relacionado à prevalência de transtornos mentais específicos, com exceção do abuso ou dependência de álcool. De fato, Koenig \& Larson ${ }^{1}$ afirmam que a relação entre denominação religiosa e prevalência de transtornos mentais tem revelado resultados contraditórios. Entretanto, dimensões da religiosidade como, por exemplo, a freqüência à Igreja e, especialmente, o envolvimento religioso, que de alguma forma indicam a intensidade da religiosidade pessoal e crença do sujeito, parecem ser mais intimamente associadas com a prevalência de transtornos mentais. Isto tem sido também comprovado por alguns estudos realizados no Brasil 14,15, mas não por outros 4,11,12. 
Freqüência de diagnóstico de transtornos do humor por religião e religiosidade.

\begin{tabular}{|c|c|c|c|c|c|c|}
\hline & \multicolumn{2}{|c|}{ Sem diagnóstico } & \multicolumn{2}{|c|}{ Com diagnóstico } & \multicolumn{2}{|c|}{ Total } \\
\hline & $\mathrm{n}$ & $\%$ & $\mathrm{n}$ & $\%$ & $\mathbf{n}$ & $\%$ \\
\hline \multicolumn{7}{|l|}{ Transtorno depressivo maior } \\
\hline \multicolumn{7}{|l|}{ Religião } \\
\hline Católico & 114 & 71,7 & 45 & 28,3 & 159 & 63,1 \\
\hline Evangélico & 39 & 67,2 & 19 & 32,8 & 58 & 23,0 \\
\hline Espírita/Outras & 9 & 60,0 & 6 & 40,0 & 15 & 5,9 \\
\hline Sem religião & 16 & 80,0 & 4 & 20,0 & 20 & 7,9 \\
\hline Total & 178 & 70,6 & 74 & 29,4 & 252 & 100,0 \\
\hline \multicolumn{7}{|l|}{ Religiosidade } \\
\hline Muito religioso & 72 & 69,2 & 32 & 30,8 & 104 & 41,6 \\
\hline Religioso & 87 & 73,7 & 31 & 26,3 & 118 & 47,2 \\
\hline Pouco/Não religioso & 18 & 64,3 & 10 & 35,7 & 28 & 11,2 \\
\hline Total & 177 & 70,8 & 73 & 29,2 & 250 & 100,0 \\
\hline \multicolumn{7}{|l|}{ Transtorno bipolar } \\
\hline \multicolumn{7}{|l|}{ Religião } \\
\hline Católico & 143 & 89,9 & 16 & 10,1 & 159 & 63,1 \\
\hline Evangélico & 54 & 93,1 & 4 & 6,9 & 58 & 23,0 \\
\hline Espírita/Outras & 13 & 86,7 & 2 & 13,3 & 15 & 5,9 \\
\hline Sem religião & 18 & 7,1 & 2 & 0,8 & 20 & 7,9 \\
\hline Total & 228 & 90,5 & 24 & 9,5 & 252 & 100,0 \\
\hline \multicolumn{7}{|l|}{ Religiosidade * } \\
\hline Muito religioso & 91 & 87,5 & 13 & 12,5 & 104 & 41,6 \\
\hline Religioso & 113 & 95,8 & 5 & 4,2 & 118 & 47,2 \\
\hline Pouco/Não religioso & 22 & 78,6 & 6 & 21,4 & 28 & 11,2 \\
\hline Total & 226 & 90,4 & 24 & 9,6 & 250 & 100,0 \\
\hline
\end{tabular}

Teste qui-quadrado: * $p=0,009$

Relacionou-se, contudo, à menor freqüência de abuso e dependência de álcool a filiação às denominações evangélicas. Também se mostraram associadas a tal freqüência reduzida uma maior freqüência a cultos e o fato de se considerar uma pessoa muito religiosa. Tais resultados provavelmente relacionam-se ao fato de as religiões de modo geral, e de as igrejas evangélicas de forma particularmente marcante, considerar o uso excessivo, abuso ou dependência de bebidas alcoólicas um comportamento condenável.

Outras possibilidades são o envolvimento e a integração num grupo social e o apoio pessoal que a pertença religiosa implica poder favorecer uma menor incidência de problemas com o álcool. Ou ainda, de forma oposta (mas resultando no mesmo efeito para a prevalência) que pessoas que se envolvam intensamente com o uso de álcool sejam de alguma forma excluídas dos grupos religiosos. A associação entre freqüência ao culto e menor prevalência de abuso/depen- dência encontrada neste trabalho vai ao encontro destas duas possibilidades. Os dados deste estudo, porém, não ajudam a decidir entre uma ou outra possibilidade.

Verificou-se também que a maior freqüência de diagnóstico de transtorno bipolar esteve significativamente associada a considerar-se uma pessoa "muito religiosa" (em relação àquelas que se consideram "religiosas"), ou considerar-se uma pessoa "pouco religiosa" ou "sem religião” (em relação àquelas que se consideram "religiosas"). Por conseguinte, associaram-se a uma maior freqüência de diagnóstico de transtorno afetivo bipolar as duas condições extremas em relação à religiosidade pessoal. Deve-se reconhecer que, embora instigantes, estes resultados são de difícil interpretação e sua análise envolve especulações pouco seguras.

É possível que pessoas sem religião sejam também aquelas com uma pior rede de apoio social ou que por seu transtorno se afastem da vida religiosa. Por outro lado, um estudo prévio 
Freqüência de diagnóstico de transtornos relacionados ao álcool e risco de suicídio por religião e religiosidade.

\begin{tabular}{|c|c|c|c|c|c|c|}
\hline & \multicolumn{2}{|c|}{ Sem diagnóstico } & \multicolumn{2}{|c|}{ Com diagnóstico } & \multicolumn{2}{|c|}{ Total } \\
\hline & $\mathbf{n}$ & $\%$ & $\mathrm{n}$ & $\%$ & $\mathbf{n}$ & $\%$ \\
\hline \multicolumn{7}{|l|}{ Risco de suicídio } \\
\hline \multicolumn{7}{|l|}{ Religião } \\
\hline Católico & 117 & 73,6 & 42 & 26,4 & 159 & 63,1 \\
\hline Evangélico & 44 & 75,9 & 14 & 24,1 & 58 & 23,0 \\
\hline Espírita/Outras & 13 & 86,7 & 2 & 13,3 & 15 & 5,9 \\
\hline Sem religião & 18 & 90,0 & 2 & 10,0 & 20 & 7,9 \\
\hline Total & 192 & 76,2 & 60 & 23,8 & 252 & 100,0 \\
\hline \multicolumn{7}{|l|}{ Religiosidade * } \\
\hline Muito religioso & 79 & 76,0 & 25 & 24,0 & 104 & 41,6 \\
\hline Religioso & 93 & 78,8 & 25 & 21,2 & 118 & 47,2 \\
\hline Pouco/Não religioso & 19 & 67,9 & 9 & 32,1 & 28 & 11,2 \\
\hline Total & 191 & 76,4 & 59 & 23,6 & 250 & 100,0 \\
\hline \multicolumn{7}{|c|}{ Abuso/Dependência de álcool } \\
\hline \multicolumn{7}{|l|}{ Religião ** } \\
\hline Católico & 145 & 91,2 & 14 & 8,8 & 159 & 63,1 \\
\hline Evangélico & 56 & 96,5 & 2 & 3,5 & 58 & 23,0 \\
\hline Espírita/Outras & 13 & 86,7 & 2 & 13,3 & 15 & 5,6 \\
\hline Sem religião & 15 & 75,0 & 5 & 25,0 & 20 & 7,9 \\
\hline Total & 229 & 90,9 & 23 & 9,1 & 252 & 100,0 \\
\hline \multicolumn{7}{|l|}{ 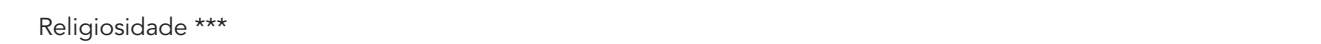 } \\
\hline Muito religioso & 100 & 96,2 & 4 & 3,8 & 104 & 41,6 \\
\hline Religioso & 107 & 90,7 & 11 & 9,3 & 118 & 47,2 \\
\hline Pouco/Não religioso & 21 & 75,0 & 7 & 25,0 & 28 & 11,2 \\
\hline Total & 228 & 91,2 & 22 & 8,8 & 250 & 100,0 \\
\hline
\end{tabular}

Teste qui-quadrado: * $\mathrm{p}=0,141 ;{ }^{\star \star \star \star} \mathrm{p}=0,002$

Teste exato de Fisher: ${ }^{\star \star} p=0,032$.

Tabela 3

Transtornos psiquiátricos e freqüência à igreja (número de vezes que foi à missa ou a um culto no último mês).

\begin{tabular}{|c|c|c|c|c|c|}
\hline Freqüência à Igreja/Transtorno & Presença do transtorno & $\mathbf{n}$ & Média (DP) & Mediana & Mínimo-máximo \\
\hline \multirow[t]{2}{*}{ Depressão maior } & Não & 158 & $4,3(5,8)$ & 2,0 & $0-30$ \\
\hline & Sim & 66 & $5,0(6,2)$ & 4,0 & $0-30$ \\
\hline \multirow[t]{2}{*}{ Agorafobia } & Não & 168 & $4,6(6,1)$ & 3,0 & $0-30$ \\
\hline & Sim & 56 & $4,1(5,4)$ & 3,0 & $0-30$ \\
\hline \multirow[t]{2}{*}{ Risco de suicídio } & Não & 170 & $4,4(5,8)$ & 3,0 & $0-30$ \\
\hline & $\operatorname{Sim}$ & 54 & $4,6(6,3)$ & 4,0 & $0-30$ \\
\hline \multirow[t]{2}{*}{ Ansiedade generalizada } & Não & 187 & $4,4(5,8)$ & 4,0 & $0-30$ \\
\hline & Sim & 37 & $4,6(6,4)$ & 2,0 & $0-30$ \\
\hline \multirow[t]{2}{*}{ Abuso/Dependência de álcool * } & Não & 204 & $4,7(6,0)$ & 4,0 & $0-30$ \\
\hline & $\operatorname{Sim}$ & 20 & $1,7(3,2)$ & 0,0 & $0-12$ \\
\hline \multirow[t]{2}{*}{ Transtorno bipolar } & Não & 201 & $4,4(5,8)$ & 3,0 & $0-30$ \\
\hline & Sim & 23 & $4,6(6,6)$ & 4,0 & $0-30$ \\
\hline
\end{tabular}

Teste de Mann-Whitney: * $p=0,0010$. 
Tabela 4

Análise multivariada com o método de seleção stepwise para obter as variáveis de religiosidade que melhor explicam a ocorrência do diagnóstico de transtorno bipolar e de abuso ou dependência de álcool.

\begin{tabular}{|c|c|c|c|c|c|}
\hline & Variáveis por passo & Níveis de comparação & Razão de risco & IC95\% & $p$ \\
\hline \multirow[t]{2}{*}{ Transtorno bipolar } & Religiosidade & Muito religioso $X$ religioso & 3,229 & $1,110-9,391$ & 0,0314 \\
\hline & & Pouco ou não religioso $X$ religioso & 6,164 & $1,728-21,984$ & 0,0051 \\
\hline \multirow[t]{2}{*}{ Abuso/Dependência de álcool } & Religiosidade & Muito religioso $X$ pouco ou não religioso & 0,134 & $0,033-0,543$ & 0,0049 \\
\hline & & Religioso X pouco ou não religioso & 0,393 & $0,127-1,211$ & 0,1039 \\
\hline
\end{tabular}

realizado por nosso grupo de pesquisa identificou que pessoas com quadros maníacos tendem a envolver-se mais com temas e conteúdos religiosos 15 . Assim, a ausência de envolvimento religioso bem como o envolvimento intenso poderiam implicar distintas dimensões da vida social e religiosa. De toda forma, os dados deste estudo, sugestivos de que os extremos de envolvimento religioso possam estar associados à maior prevalência de transtornos de humor, são ainda inconclusivos e devem ser considerados com muita cautela.

\section{Resumo}

A fim de determinar a prevalência de transtornos mentais em uma amostra de pacientes de um hospital geral e sua relação com a denominação religiosa e religiosidade, foram investigados 253 pacientes internados no Hospital das Clínicas da Universidade Estadual de Campinas por intermédio de um questionário sócio-demográfico e um instrumento para diagnóstico psiquiátrico (MINI-Plus). A maioria dos pacientes era católica (63,2\%; $n=177)$; seguidos dos evangélicos pentecostais (20,4\%; $n=57)$; dos "sem-religião" (7,5\%; $n=21)$; espiritas $(4,3 \% ; n=12)$ e protestantes históricos $(2,3 \% ; n=8)$. Consideraram-se muito religiosos $43,2 \%(n=116)$, religiosos 46,9\% ( $n=129)$, pouco religiosos $9,8 \%(n=27)$, não religiosos $1,1 \%(n=3)$. A filiação religiosa evangélica e maior freqüência a cultos relacionaram-se à menor

\section{Conclusão}

O presente estudo corrobora a noção de que a religiosidade seja uma dimensão significativa associada à prevalência de transtornos relacionados ao uso de substâncias psicoativas. Maior religiosidade pessoal, maior freqüência a cultos e a pertença a uma igreja evangélica parecem exercer, no contexto sócio-cultural brasileiro, um efeito inibidor sobre o abuso ou a dependência de álcool. A intensidade do envolvimento religioso e diferentes dimensões da religiosidade devem ser investigadas cuidadosamente em estudos futuros sobre a prevalência de transtornos mentais.

freqüência de problemas com álcool. É possível que a filiação religiosa evangélica exerça uma ação inibidora na ocorrência de transtornos relacionados ao álcool. Indivíduos muito religiosos ou pouco/nada religiosos apresentaram maior prevalência de transtorno bipolar. A dimensão intensidade da religiosidade revelou-se modestamente associada à prevalência geral dos transtornos, especialmente ao transtorno bipolar. É razoável que situações extremas (de muito ou reduzido envolvimento) relacionem-se a tal achado, relacionando tanto a busca exacerbada ou o afastamento da religiosidade com estados mentais alterados.

Religião; Transtornos Mentais; Pacientes Internados 


\section{Colaboradores}

R. E. Soeiro foi entrevistadora dos pacientes e redigiu as primeiras versões do artigo. E. S. Colombo foi entrevistadora, realizou os procedimentos de amostragem aleatória e redigiu parte das primeiras versões. M. H. F. Ferreira e P. S. A. Guimarães foram entrevistadoras, digitaram o banco de dados e redigiram parte das primeiras versões. N. J. Botega analisou os dados e participou da redação das últimas versões. P. Dalgalarrondo coordenou o trabalho, analisou os dados e participou da redação das últimas versões.

\section{Referências}

1. Koenig HG, Larson DB. Religion and mental health: evidence for an association. Int Rev Psychiatry 2001; 13:67-78.

2. Larson D, Milano MG, Lu F. Religion and mental health: the need for cultural sensitivity and synthesis. In: Okpaku SO, editor. Cultural psychiatry and mental health services. Washington DC/London: American Psychiatric Press; 1992. p. 191-210.

3. Botega NJ, Barros MBA, Oliveira HB, Dalgalarrondo P, Marin-León L. Suicidal behavior in the community: prevalence and factors associated with suicidal ideation. Rev Bras Psiquiatr 2005; 27 : 45-53.

4. Dalgalarrondo P, Marin-León L, Botega NJ, Barros MBA, Bosco-de-Oliveira H. Religious affiliation and psychiatric morbidity in Brazil: higher rates among Evangelicals and Spiritists. Int J Soc Psychiatry; submmited.

5. Pressman P, Lyons JS, Larson DB, Strain J. Religious beliefs, depression and ambulation status in elderly women with broken hips. Am J Psychiatry 1990; 147:758-60.

6. Koenig HG, George LK, Peterson BL. Religiosity and remission from depression in medically ill older patients. Am J Psychiatry 1998; 155:536-42.

7. Koenig HG, Cohen HL, Blazer DG, Pieper C, Meador KG, Shelp F, et al. Religious coping and depression in the elderly hospitalizes medically ill men. Am J Psychiatry 1992; 149:1693-700.

8. Robins PV, Fitting MD, Eastham J, Zabora J. Emotional adaptation over time in caregivers for chronically ill elderly people. Age Ageing 1990; 19:185-90.
9. Dalgalarrondo P, Banzato CEM, Botega NJ. Freqüência aumentada de esquizofrenia em pacientes evangélicos internados. J Bras Psiquiatr 2004; 53:143-52.

10. Dalgalarrondo P, Soldera MA, Correa-Filho H, Silva CM. Religião e uso de drogas por adolescentes. Rev Bras Psiquiatr 2004; 26:82-90.

11. Baptista ASD. Estudo sobre as práticas religiosas e sua relação com a saúde mental de idosos: um estudo na comunidade [Tese de Doutorado]. São Paulo: Escola Paulista de Medicina, Universidade Federal de São Paulo; 2004.

12. Floriano PJ. O perfil de idosos assistidos por uma equipe de saúde da família do Centro de Saúde de Souzas, no município de Campinas [Dissertação de Mestrado]. Campinas: Faculdade de Educação, Universidade Estadual de Campinas; 2005.

13. Amorim P. Mini International Neuropsyquiatric Interview (MINI): validação de entrevista breve para diagnóstico de transtornos mentais. Rev Bras Psiquiatr 2000; 22:106-15.

14. Volcan SM, Sousa PL, Mari JJ, Horta BL. Relationship between spiritual well-being and minor psychiatric disorders: a cross-sectional study. Rev Saúde Publica 2003; 37:440-5.

15. Dantas CR, Pavarin LB, Dalgalarrondo P. Sintomas de conteúdo religioso em pacientes psiquiátricos. Rev Bras Psiquiatr 1999; 21:158-64.

Recebido em 26/Mar/2007

Aprovado em 30/Jul/2007 